\title{
Clinical application effect of comprehensive emergency care in emergency treatment and nursing care for acute cerebral infarction with hypertension and diabetes
}

\author{
Xiaoqin $\mathrm{HU}^{1+}$, $\mathrm{Li}_{\mathrm{ZHANG}}^{2+}$, Yunfan $\mathrm{GAO}^{3 *}$ (D)
}

\begin{abstract}
To explore the emergency care method for patients suffering from acute cerebral infarction with hypertension and diabetes and its clinical effects. A total of 80 patients were selected, and were divided into the observation group and control group. The patients in the control group were given the routine emergency care, while the patients in the observation group were give the comprehensive emergency care, and the effects of clinical care of the patients in the two groups were compared. After receiving the comprehensive emergency care, the mortality rate, disability rate, recurrence rate, rescuing time, emergency triage time and NIHSS score of patients in observation group were lower than those of control group, and the differences between the two groups were of statistical significance $(\mathrm{P}<0.05)$. Giving patients suffering from acute cerebral infarction with hypertension and diabetes the comprehensive emergency care could not only save the lives of patients effectively and rapidly, reducing the mortality rate, disability rate and recurrence rate, but also effectively shorten the rescue time and clinic time of the patients, reduce the degree of neurologic impairment caused to the patients. Therefore, comprehensive emergency care is worth promoting in clinical practice.
\end{abstract}

Keywords: acute cerebral infarction; emergency care; effect of clinical care.

Practical Application: Comprehensive emergency care is worth promoting in clinical practice.

\section{Introduction}

Acute cerebral infarction is a kind of cerebrovascular disease that is common in clinical, and it will cause large damage to cerebral neuronal tissue, which will make the patient suffering from severe complications such as aphasia, hemiplegia and disturbance of consciousness after onset of this disease, and impose adverse effect on limb functions and athletic ability of the patient, posing a serious threat to the quality of life and life safety of the patient (Ye, 2019). The disease is characterized by acute onset, rapid progression of disease, poor prognosis, and the like in clinical practice, and therefore the mortality rate and disability rate of the patients are at a higher level (Niu, 2016). With the degree of aging of population in our country becoming more serious, the incidence rate of acute cerebral infarction in clinical has shown a significant upward trend. In addition, the patients with cerebral infarction are usually also suffering from hypertension and diabetes, making the disease more complicated and the clinic treatment more difficult. Relevant studies have shown that performing timely and effective emergency care for such patients in early stage is the key to reduce the mortality rate and disability rate, and has great significance for the later treatment and prognosis (Cao, 2015; Liang \& Wu, 2019). Therefore, finding the method of giving such patients timely and effective emergency care in the shortest time to get such patients out of danger as soon as possible has become a target to which the clinical workers need to make continuous efforts (Peng et al., 2016). In this paper, a total of 80 patients suffering from acute cerebral infarction with hypertension and diabetes who had received treatment in Emergency Department of our hospital over the past year were selected as the subjects for study, and the results show that giving patients comprehensive emergency care could not only reduce the mortality rate, disability rate and recurrence rate, but also effectively shorten the rescue time and clinic time of the patients and reduce the degree of neurologic impairment caused to the patients, playing an important role in later treatment and prognosis of the patients. The specific report will be given below according to the above study.

\subsection{Data and methods}

\section{General data}

In this study, the data of 80 patients suffering from acute cerebral infarction with hypertension and diabetes who had received treatment in Emergency Department of our hospital over the past year were selected. Among the patients, 48 patients were male patients, 32 patients were female patients, and their age

${ }^{1}$ Nursing Department, Suizhou Central Hospital, Affiliated Hospital of Hubei University of Medicine, Suizhou, Hubei, China

${ }^{2}$ Department of Intensive Care Unit, Suizhou Central Hospital, Affiliated Hospital of Hubei University of Medicine, Suizhou, Hubei, China

${ }^{3}$ Outpatient Department, Suizhou Central Hospital, Affiliated Hospital of Hubei University of Medicine, Suizhou, Hubei, China

*Corresponding author: gaoyunfan0123@126.com

${ }^{+}$Xiaoqin $\mathrm{Hu}$ and Li Zhang are co-first authors. 
varied from 40-79 years, with the average age of (56.45 \pm 8.87$)$ years. This study has been approved by the Ethics Committee of this hospital, and all the patients selected meet the clinical standards and are diagnosed as acute cerebral infarction with hypertension and diabetes. The family members of the patients were informed of and voluntarily accepted this study program, and cooperated to carry out this study actively. In this study, the 80 patients were divided into the observation group and control group by random grouping method, with 40 patients in each group, and the differences between the patients of the two groups in term of gender, age, clinical symptoms and other general information were not significant and were not of statistical significance $(\mathrm{P}>0.05)$, suggesting the patients of the two groups are comparable.

\section{Methods}

\subsection{Control group}

The patients in the control group were given routine emergency care: firstly, the vital signs of the patients were measured at the first time and the intravenous pathway was established rapidly; then, preliminary diagnosis was carried out by clinicians to determine the pathogeny, and corresponding emergence treatment was performed according to the actual situation; after that, the condition of the patients was observed, such as carrying out routine electrocardiograph monitoring, giving oxygen inhalation, and carrying out close monitoring on vital signs of the patients, and, for patients with ventricular fibrillation, the defibrillation was prepared (Ning, 2015).

\subsection{Observation group}

The patients in the Observation group were given comprehensive emergency care, and the specific contents of the care are as follows:

\section{Setting up a dedicated emergency care team}

Select experienced nursing personnel and physicians, as well as medical workers in Imaging Department to form dedicated Emergency Care Team. The trainings of relevant emergency care processes, especially the training of emergency rescuing skills shall be provided for medical workers according to the quality level of members of the team; and the examination about emergency care procedures shall be carried out regularly, thus to ensure the members of the Team will not make any mistake in the emergency care as far as possible, enabling the smooth performance of emergency care. In addition, the works and responsibilities of each member shall be specified clearly to let each member being aware of his responsibilities and sticking to his post, thus to finish the emergency care procedures actively and effectively, ensure the life safety and quality of life of patients, and reduce the mortality rate and disability rate of the patients.

\section{Pre-hospital first-aid and nursing}

After receiving the emergency call, the hospital shall prepare first-aid materials rapidly and organize relevant personnel to visit the patient at home within $5 \mathrm{~min}$. After arriving at the site, the nursing personnel shall cooperate with physicians to check the blood pressure, mental sense, consciousness, pulse, respiration, heart rate, pupil and activity status of arms and legs of the patient, and when the patient has cardiac arrest and respiratory arrest, carry out cardio-pulmonary resuscitation with bare hands (Jiang, 2015); measure the microamount blood glucose of the patient to check for hypoglycemia or hyperglycemia, and establish effective intravenous pathway rapidly for administration of first-aid medicine in early stage. As for transfusion, the intravenous indwelling needle shall be adopted to prevent the needle from puncturing blood vessels due to the patient's random movement and causing intravenous extravasation, and ensure the rapid input of dehydrating drugs and the timeliness and accuracy of medication; and the needle shall be placed in the site that is away from the joint and in large and straight vessel, to facilitate flowing of the fluid into the blood circulation. Since the patient with acute cerebral infarction has not had CT examination, the physician shall ask the family members about medical history, and perform corresponding pharmacy treatment according to the medical history, vital signs and symptoms of the patient. Considering that the patients suffering from acute cerebral infarction are usually companied by diabetes, the glucose solution, especially hypertonic glucose solution shall not be adopted, thus to prevent aggravating the disease. When administrating the first-aid medicines, the nursing personnel shall cooperate with the physician for first-aid, listen carefully to the verbal medical advices of the physician (ask the physician to repeat in case of any puzzle), inspect the medicines carefully and recheck the name, dose, concentration of drug, thus to prevent any mistake during administration (Sun, 2009). The electrocardiography shall be carried out for close monitoring on the vital signs and condition of the patient; for the patient with saneness, it is necessary to make the patient lying on the back with head raised slightly, untie the collar, arrange the body position of the patient reasonably, and give the patient with oxygen inhalation to keep the airway clean and prevent asphyxia of the patient; for the comatose patient, it is necessary to let the patient lie on the back with the head turning to one side, clean the excreta and vomitus in mouth and nose to prevent vomiting and asphyxia; and for the patient with severe airway obstruction, it is necessary to perform endotracheal intubation on the site to assist aspiration. Besides, the medical workers shall observe the limbs of the patient for cyanosis, and provide the patient with sufficient oxygen by using nasal catheter, thus to prevent the brain tissue from being further damaged. During the emergency care, the physicians and nursing personnel shall pay close attention to variation of blood pressure of the patient, for the high blood pressure will cause damage to the blood brain barrier, aggravating the hydrocephalus or turning the simple cerebral infarction into hemorrhagic cerebral infarction. As the compensation response to ensure blood supply of brain tissue, the patients with acute cerebral infarction are usually companied by increased intracranial pressure, and meanwhile such patients are usually accompanied by hypertension, of whom the blood pressure under normal circumstance is higher than that of normal person, and the blood pressure at onset of disease is higher than that under normal circumstance. Therefore, it is necessary to take hypertension and intracranial hypertension under control timely, thus to prevent aggravation of disease; 
for control of blood pressure of the patients with acute cerebral infarction, it is necessary to follow the principles of prudence and moderation, and select antihypertensive drugs with moderate effect (Yun, 2011). When the vital signs of the patient are basically stable, the patient shall be sent to the hospital in the shortest time if the condition permits, thus to let the patient get effective treatment as soon as possible. During transportation of the patient, it is necessary to let the patient lie on the back with the head turning to one side; carry out safety monitoring and nursing such as electrocardiograph monitoring, establishment of effective intravenous pathway, control of encephaledema, oxygen inhalation and keeping easy breathing; contact with the triage desk of the hospital to inform the receiving medical workers the specific information of the patient, thus to assist the medical workers to make preparations for rescue in advance.

\section{Emergence treatment and nursing after admission}

After arriving at the hospital, the medical workers participating in the pre-hospital first-aid shall hand over the information about disease condition, symptoms and first-aid measures and other works to relevant medical workers in the hospital; the relevant personnel participating in rescue shall carry out evaluation on the physical condition and the disease of the patient as soon as possible, measure the vital signs, perform symptomatic treatment, carry out CT examination and inform the medical personnel of each department to prepare for rescue (Zeng et al., 2019; Wang, 2019). The green channel shall be opened to enable rapid and smooth transfer of the patient to the emergency room after basic evaluation and treatment, and relevant treating physicians shall be informed to carry out rescue. In addition, the stroke treatment unit shall be prepared, clear provisions on the emergency care procedures for clinical stroke shall be made to standardize operation procedures, and a spare ward bed for thrombolysis shall be prepared to perform intravenous administration of thrombolytic drug according to the actual situation of the patient during emergency treatment and the emergency treatment process (Yan, 2019). When the patient is sent to the emergency room, the nursing personnel shall assist the physicians to establish dedicated intravenous pathway, carry out basic nursing such as oxygen inhalation and electrocardiogram monitoring, collect the blood and other specimens for clinical examination, assist the patient to complete relevant examinations, and formulate appropriate therapeutic regimen according to the actual condition of the patient; for the patient receiving thrombolysis, the nursing personnel shall strengthen the inspection, observe whether the patient has adverse conditions, and carry out close monitoring on vital signs and variation in disease condition of the patient. During the emergency treatment, the nursing personnel shall pay close attention to the blood pressure and blood glucose level of the patient, adopt mild antihypertensive drugs and drugs free of glucose to prevent aggravation of disease. In addition, it is necessary to arrange dedicated nursing personnel to inform the family members of the patient of the specific conditions of the patient and the following treatment process, and provide brief health education to let the family members have more comprehensive understanding of this kind of disease, relieve the anxiety of the family members; and provide some successful cases for the family members to increase their confidence, thus to ensure the smooth performance of the following treatment.

\subsection{Evaluation indicators}

(1) The number of deaths, number of disabled patients, number of patients suffering from recurrence in the two groups were collected and sorted out to evaluate the mortality rate, disability rate, recurrence rate of the two groups by the percentage calculation method, and carry out statistical comparison.

(2) The rescue time and emergency triage time of the patients in the two groups were collected and sorted out to evaluate the rescue time and emergency triage time of the two groups by the mean value calculation method, and carry out statistical comparison.

(3) The National Institutes of Health Stroke Scale (NISHH) (Maas et al., 2009) was adopted to evaluate the degree of neurological impairment of the patient of the two groups: 0-1 sore: normal or approximately normal; $1-4$ scores: mild stroke/ minor stroke; 5 - 15 scores: moderate stroke; 15-20 scores: moderate- severe stroke; $21-42$ scores: severe stroke. Sort out the scores of the patients of the two groups and carry out statistical comparison.

\subsection{Statistical method}

The SPSS17.0 statistical software was adopted for statistical analysis of the data in this study. Where, the enumeration data was expressed as $\mathrm{n}(\%)$, and the comparison of which among groups was tested with $\mathrm{x}^{2}$; the measurement data was expressed as $(\mathrm{x} \pm \mathrm{s})$, and the comparison of which among groups was tested with $t$ value. $\mathrm{A}=0.05$ was taken as the test criteria; $\mathrm{P}<0.05$ suggests that the difference between the two groups is of statistical significance.

\section{Results}

\subsection{Comparison of the mortality rate, disability rate and recurrence rate of the patients in the two groups}

The calculation results show that the mortality rate, disability rate and recurrence rate of the observation group is 2.5\%, 7.5\% and $12.5 \%$ respectively, while the mortality rate, disability rate and recurrence rate of the control group is $15.0 \%, 25.0 \%$ and $32.5 \%$ respectively, suggesting that the mortality rate, disability rate and recurrence rate of the observation group are significantly lower than those of the Control Group; and the value of $\mathrm{x}^{2}$ is 3.914, 4.501, 4.588 respectively, the $\mathrm{P}$ value is $0.048,0.034,0.032$ respectively, which, according the test criterion of $\alpha=0.05$, suggests that the differences of the patients in the two groups in terms of mortality rate, disability rate and recurrence rate are of statistical significance $(\mathrm{P}<0.05)$. For the specific contents, see Table 1.

\subsection{Comparison of the rescue time and emergency triage time of the patients in the two groups}

The calculation results show that the rescue time and emergency triage time of the observation group is $(1.20 \pm 0.47) \mathrm{h}$ and 
Table 1. Comparison of the mortality rate, disability rate and recurrence rate of the patients in the two groups, $\mathrm{n}(\%)$.

\begin{tabular}{ccccc}
\hline Group & Patient number $(\mathrm{n})$ & Mortality rate & Disability rate & Recurrence rate \\
\hline Observation group & 40 & $1(2.5)$ & $3(7.5)$ & $5(12.5)$ \\
Control group & 40 & $6(15.0)$ & $10(25.0)$ & $13(32.5)$ \\
$\mathrm{X}^{2}$ & & 3.914 & 4.501 & 4.588 \\
$\mathrm{P}$ & & 0.048 & 0.034 & 0.032 \\
\hline
\end{tabular}

Table 2. Comparison of the rescue time and emergency triage time of the patients in the two groups (hour, $\overline{\mathbf{X}} \pm s$ ).

\begin{tabular}{cccc}
\hline Group & $\begin{array}{c}\text { Patient number } \\
(\mathrm{n})\end{array}$ & Rescue time & $\begin{array}{c}\text { Emergency } \\
\text { triage time }\end{array}$ \\
\hline $\begin{array}{c}\text { Observation } \\
\text { group }\end{array}$ & 40 & $1.20 \pm 0.47$ & $0.57 \pm 0.25$ \\
$\begin{array}{c}\text { Control group } \\
\mathrm{t}\end{array}$ & 40 & $1.67 \pm 0.75$ & $0.85 \pm 0.24$ \\
$\mathrm{P}$ & & 3.36 & 5.132 \\
& & 0.001 & 0.000 \\
\hline
\end{tabular}

Table 3. Comparison of NISHH score of the patients in the two groups (score, $\overline{\mathbf{X}} \pm \mathrm{s}$ ).

\begin{tabular}{ccc}
\hline Group & Patient number $(\mathrm{n})$ & NISHH score \\
\hline Observation group & 40 & $7.03 \pm 4.38$ \\
Control group & 40 & $11.38 \pm 6.85$ \\
$\mathrm{t}$ & & 3.382 \\
$\mathrm{P}$ & & 0.001 \\
\hline
\end{tabular}

$(0.57 \pm 0.25) \mathrm{h}$ respectively, while the rescue time and emergency triage time of the control group is $(1.67 \pm 0.75) \mathrm{h}$ and $(0.85 \pm 0.24)$ $\mathrm{h}$ respectively, suggesting that the rescue time and emergency triage time of the observation group are significantly lower than those of the control group; and t value is 3.36 and 5.132 respectively, the $\mathrm{P}$ value is 0.001 and 0.000 respectively, which, according the test criterion of $\alpha=0.05$, suggests that the differences of the patients in the two groups in terms of rescue time and emergency triage time are of statistical significance $(\mathrm{P}<0.05)$. For the specific contents, see Table 2.

\subsection{Comparison of the degree of neurologic impairment of the patients in the two groups}

The calculation results show that the NISHH score of the observation group is $(7.03 \pm 4.38)$ scores, while the NISHH score of the control group is $(11.38 \pm 6.85)$ scores, suggesting that the NISHH score of the observation group is significantly lower than that of the control group, and the degree of neurologic impairment of the Observation Group is slighter; and $t=3.382$, $\mathrm{P}=0.001$, which, according the test criterion of $\alpha=0.05$, suggests that the difference of the patients in the two groups in terms of degree of neurologic impairment is of statistical significance $(\mathrm{P}<0.05)$. For the specific contents, see Table 3.

\section{Discussion}

The acute cerebral infarction is a common emergency and severe disease, and is characterized by acute onset, rapid progression, higher mortality rate and the like. The patients with such disease are usually accompanied by hypertension and diabetes, which will make the condition of such patients more complicated (Ma, 2016). Failure to receive appropriate emergency treatment often results in death of patient and poor prognosis, and therefore the clinical emergency care of such patient has direct impact on the clinical treatment effect of such patient (Yang et al., 2017; Yang \& Wang, 2016). In this study, the patients of the observation group were given comprehensive emergency care: firstly, the dedicated emergency skills training were provided for the medical workers taking responsible for this group, and the works and responsibilities of each member were specified to avoid the adverse effect on effect of later treatment as a result of unclear division of works and responses; then timely and effective pre-hospital first-aid and nursing measures were implemented to ensure the smooth performance of rescue; after arriving at the hospital, the handover of work was done in a timely manner, and an reasonable and effective emergency care program was formulated according to the disease condition and basic information of the patient, thus to reduce the emergency treatment time of the patient, capture the optimal time for treatment, ensuring the patient can receives effective treatment within the shortest period (Wang \& Ma, 2019; Cao, 2016). The results show that the mortality rate, disability rate, recurrence rate, rescue time, emergency triage time and NIHSS score of the observation group are significantly lower than those of the control group, and the differences between the two groups are of statistical significance $(\mathrm{P}<0.05)$.

Above all, giving comprehensive emergency care to the patients suffering from acute cerebral infarction with hypertension and diabetes could effectively shorten the rescue time and emergency triage time of the patients, let the patients have timely and effective treatment, thus to reduce the mortality rate, disability rate and recurrence rate of the patients, reduce the degree of neurological impairment of the patients. Comprehensive emergency care plays a significant role in the later treatment and prognosis, and therefore is worth promoting in clinical practice.

\section{References}

Cao, G. P. (2015). Analysis on clinical first aid and nursing recovery of the patients with Acute Cerebral Infarction. Chinese Continuing Medical Education, 7(21), 228-229.

Cao, M. Q. (2016). Impact of optimized emergency nursing procedures on the emergency efficiency and life quality of the patients with Acute Cerebral Infarction. Journal of Qilu Nursing, 22(6), 33-35.

Jiang, Y. L. (2015). Effect of emergency care processes in emergency treatment of patients with Acute Cerebral Infarction. World Latest Medicine Information, 28, 228-229. 
Liang, Z. Q., \& Wu, Y. F. (2019). A study on the emergency treatment effects of optimizing emergency nursing procedure on the patients with Acute Cerebral Infarction. Chinese Journal of General Practice, 17(4), 682-685.

Ma, Y. (2016). Research on clinical emergency treatment and nursing of patients with Acute Cerebral Infarction. Clinical Medicine, 29(36), 208.

Maas, M. B., Furie, K. L., Lev, M. H., Ay, H., Singhal, A. B., Greer, D. M., Harris, G. J., Halpern, E., Koroshetz, W. J., \& Smith, W. S. (2009). National Institutes of health stroke scale score is poorly predictive of proximal occlusion in Acute Cerebral Ischemia. Stroke, 40(9), 2988-2993. http://dx.doi.org/10.1161/STROKEAHA.109.555664. PMid:19608992.

Ning, L. Y. (2015). Influence of emergency care processes on effects of emergency treatment of patients with Acute Cerebral Infarction. Practical Journal of Cardiac Cerebral Pneumal and Vascular Disease, 23(1), 152-153.

Niu, J. (2016). Clinical emergency treatment and nursing of patients with Acute Cerebral Infarction. China Practical Medicine, 11(8), 221-222.

Peng, B., Wu, D. Y., \& Sun, J. (2016). The correlation between mirnas levels and collateral pathway in patients with Acute Cerebral Infraction. Apoplexy and Nervous Diseases, 33(2), 79-80.

Sun, X. L. (2009). Experience of pre-hospital first aid and nursing for Acute Cerebrovascular Accident. China Practical Medicine, 4(14), 191-192.
Wang, C., \& Ma, P. Y. (2019). Clinical effects of emergency care processes in emergency treatment and nursing of patients with Acute Cerebral Infarction. World Latest Medicine Information, 19(48), 280-282.

Wang, H. W. (2019). A study on the effect of full-process optmized emergency nursing in the rescue of the patients with Acute Cerebral Infarction. Practical Clinical Nursing E-magazine, 4(42), 4-6.

Yan, X. (2019). Impacts of in-hospital integrated emergency nursing mode on the treatment effects of the patients with Acute Cerebral Infarction. Guide of China Medicine, 17(22), 227-228.

Yang, H., Huang, G. X., \& Zeng, J. (2017). Observation on application effect of optimal emergency care processes in rescue of patients with Acute Myocardial Infarction. Practical Journal of Clinical Medicine, 14(2), 83-85.

Yang, Q., \& Wang, P. Q. (2016). The application effect of emergency nursing procedures on the nursing of the patients with Acute Cerebral Infarction. Chinese Journal of Modern Nursing, 22(13), 1834-1836.

Ye, W. J. (2019). Observation on clinical effects of emergency care processes in emergency treatment and nursing of patients with Acute Cerebral Infarction. Psychologies, 14(20), 151.

Yun, P. (2011). Experience of first aid and nursing for Acute Cerebrovascular Accident. Chinese General Practice Nursing, 6, 2409-2410.

Zeng, Y. H., Liu, Y., \& Chen, X. L. (2019). clinical effects of emergency care processes in emergency treatment and nursing of patients with Acute Cerebral Infarction. Health Vocational Education, 37(2), 155-156. 